\title{
Fully Passive Conductive-Bridging Solid State RF Switch
}

\author{
M.P. Jayakrishnan ${ }^{(1)}$, Arnaud Vena ${ }^{(2)}$, Brice Sorli ${ }^{(2)}$ and Etienne Perret ${ }^{(1,3)}$ \\ (1) Université Grenoble Alpes - LCIS, 50, rue de Laffemas, BP 54, Valence 26902, France. \\ (2) Institut d'Electronique du Sud, Université de Montpellier 2, Montpellier 34095, France. \\ (3) Institut Universitaire de France, Paris 75005, France \\ Email:jkmpjk@gmail.com, etienne.perret@lcis.grenoble-inp.fr
}

\begin{abstract}
This study reports the design and experimental results of a fully-passive solid-state RF switch based on the Conductive Bridging Memory Technology, popularly known as CBRAM. The developed device is a shunt mode RF switch based on a Metal-Insulator-Metal (MIM) structure with Copper - Nafion - Aluminum switching layers on a Coplanar Waveguide (CPW) transmission line, operational in the $\mathrm{DC}$ to $3 \mathrm{GHz}$ range. $\mathrm{DC}$ pulses in the range $+12 \mathrm{~V}$ to $-20 \mathrm{~V}$ are used to operate the switch. The design is initially simulated using the FEM based CST microwave Studio and then realized and validated on a low cost FR4 substrate, and without using any sophisticated clean room technology.
\end{abstract}

\section{Keywords: CBRAM, Memristor, RF-Switch}

\section{Introduction}

Since 1971 when Leon Chua [1] pointed out the evidence for the existence of a fourth basic circuit element - the Memristor, and when Prof. Michael Kozicki [2] of Arizona State University demonstrated the memory switching properties of Memristive devices in 1996, the quest for development of a true solid state switch has begun. With advances in the Digital signal processing and miniaturization of circuits, the urge for a true solid state and passive switch has been intensified in the RF Engineering field. This stimulus has led to extensive research all over the world in this field. The currently available state of the art solutions are the MEMS RFswitches [3], which are operational from $\mathrm{DC}$ to $\mathrm{THz}$ range. But the MEMS switch has the drawback that it needs a constant power supply to maintain the state, which adds to the power budget of the device. The Memristive switch is a promising technology which solves the problem of requiring a constant power supply, thus reducing the overall power consumption of the device. Few Memristive RF-switching devices are reported based on PCM [4], CBRAM [5, 6] etc., in the recent years.

In this paper we report a simple and reliable solution for RF-Switch based on the CBRAM technology using simple fabrication steps and without the need for a clean room environment. The results show acceptable performance and good repeatability, which makes this concept a promising set forth.

\section{Background}

The CBRAM RF switch is based on a Metal-InsulatorMetal structure as shown in Fig. 1 where the Insulator layer is a solid electrolyte like common synthetic resin $[6,7]$ and one of the electrode is an electrochemically active metal like copper or silver and the other is a relatively inert metal like aluminum or gold. The switching action is obtained by applying electric field of sufficient voltage to establish a metallic link between the two electrodes through the electrolyte, and by dissolving this link using an electric field of opposite polarity, to establish the SET and RESET states respectively, as shown in the Fig. 1. The switch thus formed is fully passive, in sense, that it does not require an external power supply to maintain the state.

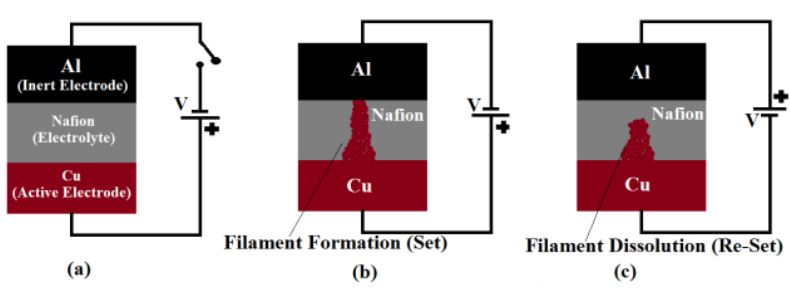

Fig. 1. (a) The Conductive Bridging MIM structure, (b) SET state, (c) RE-SET state

\section{Design and Development}

The design and development of the RF Switch is done using basic in house lab facilities and without utilizing any sophisticated clean room technology.

The Fig. 2 shows the topology of the shunt mode RF switch on a $50 \Omega \mathrm{CPW}$ transmission line. The design was first simulated using the FEM based CST Microwave studio. The operation of the switch is as follows. The two ground planes of the CPW line are interconnected using a thin line (Shunt Line) as shown in the Fig. 2, this line and the signal line sandwiches a thin $(2 \mu \mathrm{m})$ layer of Nafion, which acts as a solid electrolyte. The switching action is observed in this MIM structure formed by the CopperNafion-Aluminum layers. When a conductive filament is formed between the signal line and the ground, the RFEnergy from the port 1 is short circuited to the ground and isolates port 1 from port 2 this defines the OFF state of the RF Switch (i.e. SET mode) and when the conductive 
filament between the signal line and the ground is dissolved using a voltage pulse, the RF-energy from port 1 reaches the port 2, which defines the ON state of the RF switch (RESET state).

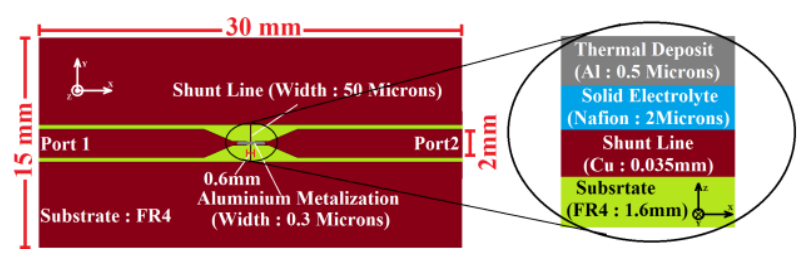

Fig. 2. The topology of the shunt mode RF Switch and the cross section view of the MIM structure, with the thickness of each layer.

The copper trace was first printed on the FR4 substrate using ordinary photolithography technique, the thickness of the copper trace is $35 \mu \mathrm{m}$. The printed trace is then coated with Nafion perflourinated resin solution supplied by Sigma Aldrich using a programmable spin coater at a rate of $500 \mathrm{rpm}$ for 30 seconds to achieve a $2 \mu \mathrm{m}$ thick layer of Nafion. The layer thickness is measured using the Dektak-150 Surface Profiler. The inert electrode (Aluminum) is formed using a Nickel mask by depositing Aluminum by thermal evaporation. The thickness of the inert electrode is $0.5 \mu \mathrm{m}$. The photograph of the fabricated device is shown in the Fig. 3.

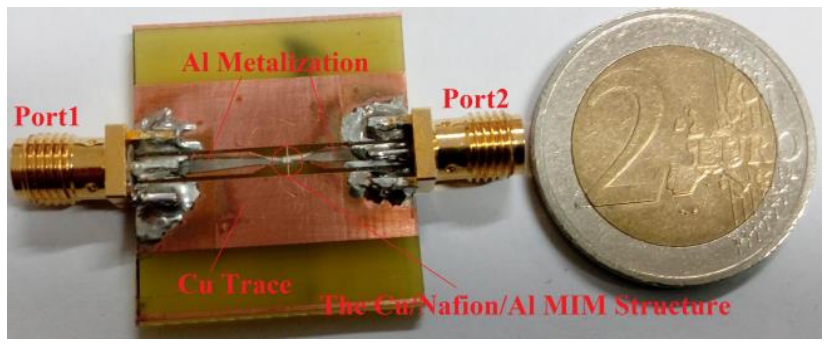

Fig. 3. Photograph of the fabricated RF switch.

The switch is operated using DC pulses of $+12 \mathrm{~V} / 500 \mu \mathrm{A}$ for setting and $-20 \mathrm{~V} / 200 \mathrm{~mA}$ for reset. The setting and resetting of the switch was done on the Cascade Microtech-PM5 Probe station using DC probes and the set-reset pulses were generated using the Kethley-2400 source-meter controlled with Labview Software. The SetReset pulses are shown in the Fig. 4. During the SET process, the voltage pulse follows the path marked by the dashed orange line if in case the switch is not SET; otherwise the voltage drops to zero to indicate a short circuit, as shown by the purple trace.

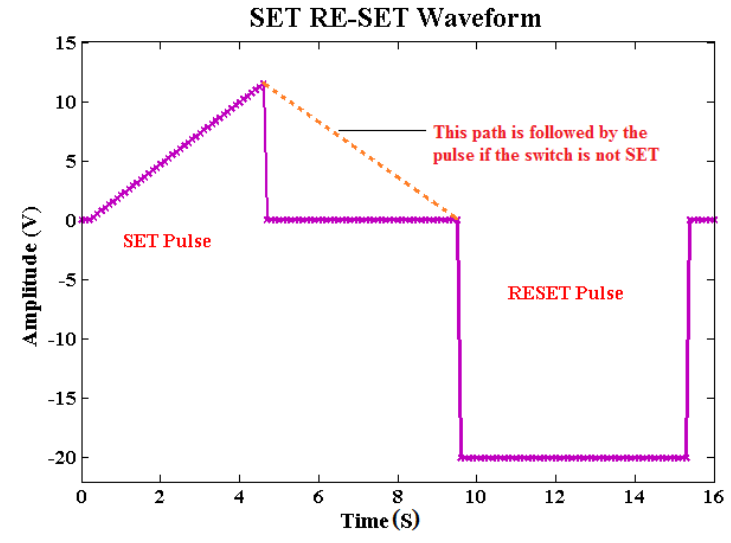

Fig. 4. The SET RE-SET pulses used to operate the switch.

The RF-measurements of the switch was done using the Agilent ENA E5061B and the S-parameters of the switch are shown in the Fig. 5, for three consecutive switching cycles.

\section{Transmission Response of the Switch}

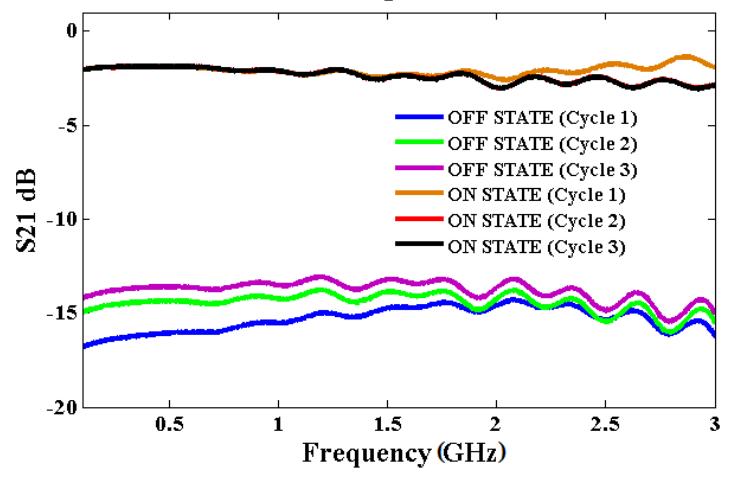

Port Reflection Response of the Switch

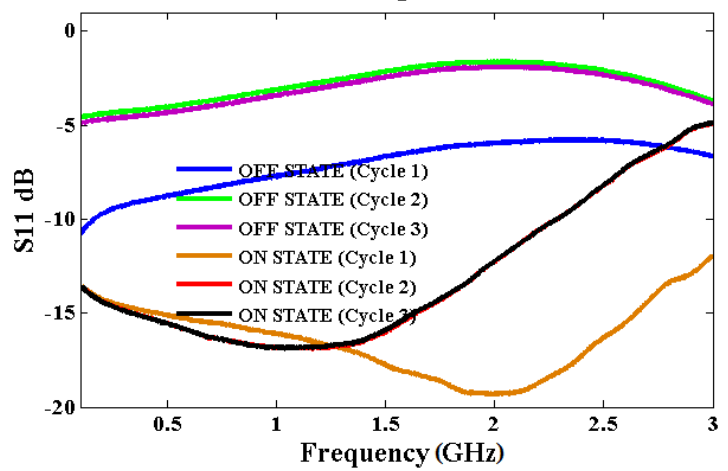

Fig. 5. The S-parameters of the fabricated switch.

\section{Results and Discussion}

The switch shows a linear behavior in the ON and OFF state as shown in the Fig. 5, the ON and OFF state DC resistance of the switch is measured to be in the range 10 to $20 \Omega$ and 1 to $10 \mathrm{G} \Omega$ respectively. In the $\mathrm{ON}$ and $\mathrm{OFF}$ 
state the $\mathrm{S} 21$ is better than $-2 \mathrm{~dB}$ and is lower than $-14 \mathrm{~dB}$, respectively, in the entire bandwidth. The measured resistance and the RF-parameters correspond to the complete structure including the CPW feed lines and the SMA connectors, and also have the effects of FR4 which is not a very efficient RF substrate. The real performance of the CBRAM switch could be understood only after applying appropriate de-embedding to these results, and in practice would be more superior to these preliminary results.

\section{Conclusion}

This study reports and proves the feasibility of a low cost solid state passive RF switch with acceptable performance and mechanical stability. The ease of production and compactness enables the developed device to be used in reconfigurable filters, phase shifters, RFID etc. The mechanical stability and rugged nature of the device make it suitable for highly sophisticated devices like satellites. The switch does not add to the weight or space of the RF circuitry but could be directly incorporated in the available space. As there are no magnetic or moving parts the switch is safe to electromagnetic interferences and one could not hack the present state of the switch, unless by directly probing the device. This study apparently assures that in near future we could achieve a fully printed RFswitch technology, on RF substrates, by printing consecutive layers of the conductors and the solid electrolyte.

\section{Acknowledgements}

The authors are grateful to Grenoble Institute of Technology, Université Grenoble Alpes and Institut Universitaire de France for supporting this project.

\section{References}

1. Leon O. Chua, "Memristor-The Missing Circuit Element", IEEE Trans. Circuit Theory, vol. 18, no. 5, pp. 507-519, September 1971.

2. Michael N. Kozicki, "Programmable Metallization Cell Structure and Method of Making Same", U.S. Patent 5,761,115, Jun.2, 1998.

3. Charles L. Goldsmith et al., "Performance of Low-Loss RF MEMS Capacitive Switches", IEEE Microw. Guided Wave Lett. , vol. 8, no. 8, pp. 269-271, August 1998.

4. Nabil El-Hinnawy et al., "Low-loss latching microwave switch using thermally pulsed non-volatile chalcogenide phase change materials", Appl. Phys. Lett., vol. 105, 2014. Doi: http://dx.doi.org/10.1063/1.4885388.
5. J. Nessel, Hinckley and Richard Lee, "Chalcogenide Nanoionic-Based Radio Frequency Switch", U.S. Patent US 7,923,715 B2, Apr. 12, 2011.

6. E. Perret et al.," Realization of a Conductive Bridging RF Switch Integrated onto Printed Circuit Board", Progress in Electromagn. Res., vol. 151, pp. 9-16, 2015.

7. Jiaying Jian et al., "Study and design of resistive switching behaviors in PMMA based conducting bridge random access memory (CBRAM) devices", Microsyst. Techno. , 2015. Doi: 10.1007/s00542-015-2754-6. 